\title{
Body weight definitions for evaluating a urinary diagnosis of acute kidney injury in patients with sepsis
}

Shinshu Katayama* ${ }^{*}$, Kansuke Koyama, Yuya Goto, Toshitaka Koinuma, Ken Tonai, Jun Shima, Masahiko Wada and Shin Nunomiya

\begin{abstract}
Background: We hypothesized that the use of actual body weight might lead to more frequent misdiagnosis of acute kidney injury (AKI) than when ideal body weight is used in underweight and/or obese patients. We examined which definition of body weight is most effective in establishing a urinary diagnosis of AKI in septic patients.

Methods: Consecutive patients aged $\geq 20$ years admitted to the intensive care unit of a university hospital between June 2011 and December 2016 were analyzed. Sepsis was defined in accordance with the Sepsis-3 criteria. AKI was defined as a urinary output of $<0.5 \mathrm{~mL} / \mathrm{kg} / 6 \mathrm{~h}$ during intensive care unit stay. Patients were divided into one of four body mass index-based classes. The severity of illness and 90-day mortality were compared across the body mass index subgroups in patients diagnosed using the actual body weight or ideal body weight.

Results: Of 5764 patients, 569 septic patients were analyzed. One hundred and fifty-three (26.9\%) and 140 (24.6\%) patients were diagnosed as having AKI using actual body weight and ideal body weight, respectively. There were no significant differences in the severity of illness among these groups. Also, 90-day mortality did not differ significantly among these groups. According to body mass index, 90-day mortality significantly differed in patients diagnosed using their actual body weights (underweight vs. normal vs. overweight vs. obese: $76.7 \%$ vs. $39.5 \%$ vs. $26.0 \%$ vs. $35.7 \%, P=0.033)$.

Conclusion: Generally, using actual body weight to calculate the weight-adjusted hourly urine output for diagnosing AKI increased the sensitivity compared to ideal body weight, irrespective of the severity of illness in septic patients. Delayed diagnosis, however, was more common among underweight patients in this situation, and clinicians should be cautious when diagnosing urinary AKI using actual body weight.
\end{abstract}

Keywords: Acute kidney injury, Body mass index, Cystatin C, Ideal body weight, Intensive care unit, Sepsis

\section{Background}

Acute kidney injury (AKI) is a major complication in sepsis, and is associated with a high mortality rate. Nearly half of the patients in the intensive care unit (ICU) develop AKI, and the mortality rate among these patients ranges from $30 \%$ to $50 \%[1,2]$. Early recognition of AKI is therefore important as it allows for early treatment initiation and better prognostication of the clinical course in patients with sepsis.

\footnotetext{
*Correspondence: shinsyu_k@jichi.ac.jp

Division of Intensive Care, Department of Anesthesiology and Intensive Care Medicine, Jichi Medical University School of Medicine, 3311-1, Yakushiji, Shimotsuke, Tochigi 329-0498, Japan
}

The definition of AKI is based on absolute or relative changes in serum creatinine levels and the weightadjusted hourly urine output [3]. The weight-adjusted hourly urine output is closely associated with early onset of AKI and high mortality rates [4-6]. To date, however, the precise body weight definition that should be used for establishing a urinary diagnosis of AKI remains unclear. For example, in patients with obesity or weight loss, there is a substantial difference between the actual body weight $(\mathrm{ABW})$ and ideal body weight (IBW), which can lead to under- or over-diagnosis of AKI.

Only one study [7] has evaluated the influence of body weight on the urinary diagnosis of AKI thus far; they 
reported that $A B W$ was more sensitive but less specific than IBW. In contrast, in acute respiratory distress syndrome (ARDS) [8] and obese patients [9], IBW is recommended over $A B W$ because the sizes of the vital organs are more closely related to IBW than to $A B W$. In this regard, we hypothesized that IBW is more useful in terms of evaluating renal function and establishing a urinary diagnosis of $\mathrm{AKI}$ than $\mathrm{ABW}$ in heterogeneous patient populations.

Therefore, in this study, we investigated the influence of body weight definitions on the urinary diagnosis of AKI in septic patients who were at risk of AKI. In addition, we evaluated the severity of illness and 90-day mortality for each body weight type determined according to each patients' body mass index (BMI).

\section{Methods}

\section{Study design and setting}

This was a single-center, retrospective, observational study that was conducted in a 14-bed general ICU of a university hospital (Tochigi, Japan). Clinical decisions were made at the discretion of the attending ICU physicians. The Institutional Research Ethics Committee of Jichi Medical University Hospital approved this study and waived the need for informed consent because of the retrospective nature of the study (No. 16-116).

\section{Participants}

Data for consecutive patients admitted to the ICU between June 2011 and December 2016 were screened. Patients were included in the study if they were $\geq 20$ years of age, admitted to the ICU for a minimum of 6 continuous hours, underwent hourly urine output measurements, and were diagnosed with sepsis according to the Third International Consensus Definitions for Sepsis and Septic Shock (Sepsis-3) [10]. Patients with end-stage renal failure who were on chronic dialysis, those with inadequate hourly urine output measurements, and those with missing data for body weight and/or height were excluded.

\section{Definitions}

The urinary diagnosis and staging of AKI were based on the Kidney Disease: Improving Global Outcomes (KDIGO) criteria [1]. A urinary diagnosis of AKI is established via 2 methods: the use of ABW or IBW for the calculation of weight-adjusted hourly urine output; in this study, these factors are indicated as AKI [ABW] and AKI [IBW], respectively. ABW was measured on or around the day of ICU admission. IBW was calculated using the Devine formula as follows [11]:

Males: IBW $(\mathrm{kg})=50 \mathrm{~kg}+0.91$ * (Height $[\mathrm{cm}]-152.4)$.

Females: IBW $(\mathrm{kg})=45.5 \mathrm{~kg}+0.91 *$ (Height $[\mathrm{cm}]-152.4)$.
Patients were classified into one of four BMI classes: underweight $(\mathrm{BMI}<18.5)$, normal $(18.5 \leq \mathrm{BMI}<25)$, overweight $(25 \leq \mathrm{BMI}<30)$, and obese $(30 \leq \mathrm{BMI})$. Chronic kidney disease (CKD) was defined as an estimated glomerular filtration rate (eGFR) of $<60 \mathrm{~mL} / \mathrm{min} / 1.73 \mathrm{~m}^{2}$ [12] Overt disseminated intravascular coagulation was defined according to the criteria of the International Society on Thrombosis and Haemostasis [13]. The baseline serum creatinine value was defined as the stable value observed over the year prior to hospital admission. For patients whose baseline serum creatinine values were unknown, we used the Modification of Diet in Renal Disease (MDRD) equation, and assumed a baseline eGFR of $75 \mathrm{~mL} / \mathrm{min} / 1.73 \mathrm{~m}^{2}$ [14].

\section{Data collection}

In this study, we collected the urine output data during the ICU stay (maximum of 7 days). The following information was collected for all patients: age; sex; body weight; body height; premorbid creatinine level; infection site (intra-cranial, head and neck, thoracic, abdominal, urinary tract, skin and soft tissue, catheter related blood stream infection, or others); ischemic heart disease, chronic heart failure, hypertension, arrhythmia, chronic obstructive pulmonary disease, cerebrovascular accident, diabetes mellitus, hepatic disease, or chronic kidney disease; an immunocompromised state; use of aminoglycoside or vancomycin; the Simplified Acute Physiology Score II (SAPS-II) [15]; and the Sequential Organ Failure Assessment (SOFA) score [16]. We also recorded the presence of septic shock, overt disseminated intravascular coagulation, the requirement of mechanical ventilation, duration of mechanical ventilation and ICU stay, and mortality rate at 90 days. In our ICU, hourly urine output measurements are made for almost all patients, except for patients undergoing renal replacement therapy (RRT) whose urine outputs are measured every $3 \mathrm{~h}$. Therefore, in the case of RRT patients, we calculated the mean hourly urine output. The highest values of serum creatinine and cystatin $\mathrm{C}$ levels were mostly realized in the first $48 \mathrm{~h}$ after admission to the ICU.

\section{Statistical analyses}

Variables were compared between the two groups using Fisher's exact test, Pearson's chi-squared test, and the Mann-Whitney $U$ test as appropriate. The Wilcoxon/ Kruskal-Wallis test was used to compare variables among BMI classes. The Kaplan-Meier method and logrank test were used to calculate the cumulative mortality rate. The risk ratio of mortality was calculated using a proportional hazard model. Multiple regression lines were analyzed to evaluate the relationships between cystatin $\mathrm{C}$ and creatinine values across BMI classes. All analyses were performed using the JMP 13 software 
program (SAS Institute Inc., Cary, NC, USA). The data are presented as medians and interquartile ranges (25th-75th percentiles) or percentages. $P$-values less than 0.05 were considered statistically significant.

\section{Results}

\section{Enrollment and baseline characteristics}

During the study period, 5764 patients were admitted to the ICU. Among these, 651 were diagnosed with sepsis; furthermore, 49 patients who were receiving chronic dialysis owing to end-stage renal failure, 3 with inaccurate urine measurements (i.e., bladder irrigation), and 30 with missing body weight and height measurements were excluded. Thus, data for a total of 569 patients were analyzed in this study (Fig. 1).

The characteristics of the analyzed patients are summarized in Table 1. Herein, 153 (26.9\%) and 140 (24.6\%) patients were diagnosed with $\mathrm{AKI}[\mathrm{ABW}]$ and $\mathrm{AKI}[\mathrm{IBW}]$, respectively. The mean (range) $\mathrm{BMI}$ of the AKI [ABW] and AKI $[\mathrm{IBW}]$ groups were $23.6(21.1-26.6)$ and 23.0 (20.1-25.5), respectively. Baseline characteristics were comparable between the groups. Besides, the severity of illness between groups did not differ significantly, and 90day mortality was $38.4 \%$ in the AKI [ABW] group and $42.3 \%$ in the AKI [IBW] group (Table 1).

In the AKI $[\mathrm{ABW}$ ] group, 129 (84.3\%) patients were diagnosed with AKI based on the level of serum creatinine, and $24(15.7 \%)$ without AKI. On the other hand, in the AKI [IBW] group, $122(87.1 \%)$ patients were diagnosed with AKI and 18 (12.9\%) without AKI based on the level of serum creatinine.

\section{Urinary diagnosis of $A K I$ and staging with $A B W$ and IBW}

In the AKI $[\mathrm{ABW}]$ group, $51(9.0 \%)$ patients had stage 1 disease, $38(6.7 \%)$ stage 2 , and 64 (11.3\%) stage 3 . In the AKI [IBW] group, 45 (7.9\%) patients had stage 1 disease, $32(5.6 \%)$ stage 2 , and $63(11.1 \%)$ stage 3 . The concordance rate between the groups was $92.4 \%(n=526)$. When using $\mathrm{ABW}$ to diagnose $\mathrm{AKI}, 31$ patients (5.4\%) were classified into higher AKI stages, while 12 patients (2.2\%) were classified into lower AKI stages than those diagnosed using IBW (Table 2).

\section{Relationship between urinary diagnosis of AKI and BMI}

Table 3 shows the relationship between urinary diagnosis of AKI and BMI. The AKI [ABW] group included 12 underweight patients $(7.8 \%), 83$ patients $(54.2 \%)$ with normal weight, 40 overweight patients (26.1\%), and 18 obese patients (11.8\%). The AKI [IBW] group comprised 19 underweight patients (13.6\%), 80 patients (57.1\%) with normal weight, 28 overweight patients (20.0\%), and 13 obese patients (9.3\%).

In the AKI $[\mathrm{ABW}]$ group, the underweight BMI subgroup demonstrated significantly higher mortality rates

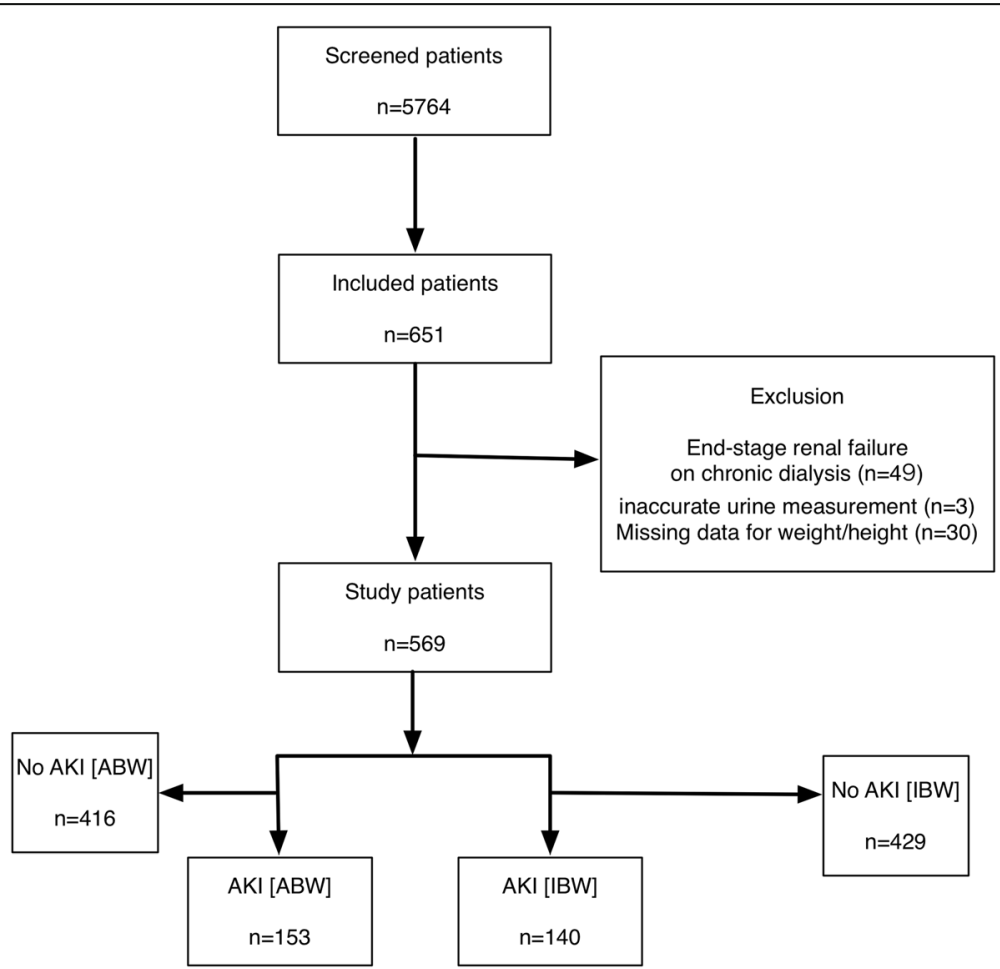

Fig. 1 Flow chart of this study. ABW, actual body weight; AKI, acute kidney injury; BW, body weight; CKD, chronic kidney disease; IBW, ideal body weight 
Table 1 Patient characteristics

\begin{tabular}{|c|c|c|c|c|}
\hline \multirow[t]{2}{*}{ Characteristics } & \multirow{2}{*}{$\begin{array}{l}\text { Total } \\
n=569\end{array}$} & \multirow{2}{*}{$\begin{array}{l}\text { AKI [ABW] } \\
n=153\end{array}$} & \multirow{2}{*}{$\begin{array}{l}\text { AKI [IBW] } \\
n=140\end{array}$} & \multirow[t]{2}{*}{$P$-value } \\
\hline & & & & \\
\hline Age, years & $69(59-78)$ & $69(59-78)$ & $69(59-78)$ & 0.998 \\
\hline Male sex, No. & 305 (53.6\%) & 89 (58.2\%) & 89 (63.6\%) & 0.344 \\
\hline Body weight, kg & $57(48-66)$ & $59(52-70)$ & $58(50-68)$ & 0.293 \\
\hline Height, cm & $158(150-166)$ & $160(152-167)$ & $160(154-167)$ & 0.452 \\
\hline $\mathrm{BMI}, \mathrm{kg} / \mathrm{m}^{2}$ & $22.5(19.7-25.3)$ & $23.6(21.1-26.6)$ & $23.0(20.1-25.5)$ & 0.082 \\
\hline Infection site, No. & & & & 0.999 \\
\hline CNS & $6(1.0 \%)$ & $0(0 \%)$ & $0(0 \%)$ & \\
\hline Thorax & 128 (22.5\%) & 37 (24.2\%) & $33(23.6 \%)$ & \\
\hline Abdomen & 301 (52.9\%) & $72(47.1 \%)$ & 64 (45.7\%) & \\
\hline Head and neck & $25(4.4 \%)$ & $1(0.7 \%)$ & $1(0.7 \%)$ & \\
\hline Soft tissue & $32(5.6 \%)$ & $14(9.2 \%)$ & $13(9.3 \%)$ & \\
\hline UTI & $25(4.4 \%)$ & $2(1.3 \%)$ & $2(1.4 \%)$ & \\
\hline CR-BSI & $5(0.9 \%)$ & $4(2.6 \%)$ & $3(2.1 \%)$ & \\
\hline Other & $49(8.6 \%)$ & $23(15.0 \%)$ & $24(17.1 \%)$ & \\
\hline Unknown premorbid creatinine, No. & $254(44.6 \%)$ & $58(37.9 \%)$ & $54(38.6 \%)$ & \\
\hline Premorbid creatinine, mg/dL & $0.75(0.60-0.96)$ & $0.82(0.63-1.09)$ & $0.83(0.69-1.18)$ & 0.756 \\
\hline \multicolumn{5}{|l|}{ Comorbidities, No. } \\
\hline CKD & $158(27.8 \%)$ & $58(61.1 \%)$ & $54(61.4 \%)$ & 0.810 \\
\hline IHD & $53(9.3 \%)$ & $18(11.8 \%)$ & $14(10.0 \%)$ & 0.629 \\
\hline $\mathrm{CHF}$ & $50(8.8 \%)$ & $21(13.7 \%)$ & $16(11.4 \%)$ & 0.554 \\
\hline Hypertension & $273(48.0 \%)$ & $84(54.9 \%)$ & $74(52.9 \%)$ & 0.726 \\
\hline Arrhythmia & $57(10.0 \%)$ & $23(15.0 \%)$ & $20(14.3 \%)$ & 0.857 \\
\hline COPD & $32(5.6 \%)$ & $6(3.9 \%)$ & $7(5.0 \%)$ & 0.654 \\
\hline CVA & $62(10.9 \%)$ & $21(13.7 \%)$ & $16(11.4 \%)$ & 0.554 \\
\hline $\mathrm{DM}$ & $145(25.5 \%)$ & $47(30.7 \%)$ & $43(30.7 \%)$ & 0.999 \\
\hline Immune dysfunction & $171(30.0 \%)$ & $54(35.3 \%)$ & $51(36.4 \%)$ & 0.840 \\
\hline Hepatic disease & $53(9.3 \%)$ & $18(11.8 \%)$ & $18(12.9 \%)$ & 0.776 \\
\hline Aminoglycoside use & $8(1.4 \%)$ & $3(2.0 \%)$ & $3(2.1 \%)$ & 0.913 \\
\hline Vancomycin use & $139(24.4 \%)$ & $55(36.0 \%)$ & $49(35.0 \%)$ & 0.866 \\
\hline Septic shock & $247(43.4 \%)$ & $87(56.9 \%)$ & $81(57.9 \%)$ & 0.864 \\
\hline ISTH DIC & $125(22.5 \%)$ & $55(36.7 \%)$ & $54(39.4 \%)$ & 0.632 \\
\hline SOFA score & $7(4-9)$ & $9(7-11)$ & $10(7-12)$ & 0.617 \\
\hline SAPS ॥ & $50(39-62)$ & $63(52-77)$ & $64(51-77)$ & 0.760 \\
\hline Urinary diagnosis of AKI, No. & & & & 0.852 \\
\hline Stage 1 & & $51(9.0 \%)$ & $45(7.9 \%)$ & \\
\hline Stage 2 & & $38(6.7 \%)$ & $32(5.6 \%)$ & \\
\hline Stage 3 & & $64(11.2 \%)$ & $63(11.1 \%)$ & \\
\hline Mechanical ventilation, No. & $462(81.2 \%)$ & 139 (90.9\%) & $124(88.6 \%)$ & 0.521 \\
\hline Days of mechanical ventilation & $7(5-12)$ & $8(6-15)$ & $9(6-16)$ & 0.957 \\
\hline Days of ICU stay & $8(5-12)$ & $10(7-16)$ & $10(6-16)$ & 0.862 \\
\hline 90-day mortality & $19.0 \%$ & $38.4 \%$ & $42.3 \%$ & 0.624 \\
\hline
\end{tabular}

$A B W$ actual body weight, $A K I$ acute kidney injury, $B M I$ body mass index, $B W$ body weight, $C H D$ chronic heart disease, $C K D$ chronic kidney disease, $C N S$ central nervous system, COPD chronic obstructive pulmonary disease, CR-BSI catheter-related blood stream infection, CVA cerebrovascular accident, DIC disseminated intravascular coagulation, DM diabetes mellitus, IBW ideal body weight, ICU intensive-care unit, ISTH International Society on Thrombosis and Hemostasis, SAPS II sequence assessment of physiological score II, SOFA Sequential Organ Failure Assessment, UTI urinary tract infection 
Table 2 Acute kidney injury diagnoses and staging

\begin{tabular}{lllllll}
\hline & & \multicolumn{3}{l}{ AKI [ABW] } & & \\
\cline { 3 - 7 } & & Stage 0 & Stage 1 & Stage 2 & Stage 3 & Total \\
\hline AKI [IBW] & Stage 0 & 406 & 20 & 2 & 1 & 429 \\
& & $71.4 \%$ & $3.5 \%$ & $0.4 \%$ & $0.2 \%$ & $75.5 \%$ \\
& Stage 1 & 8 & 29 & 8 & 0 & 45 \\
& $1.4 \%$ & $5.1 \%$ & $1.4 \%$ & $0 \%$ & $7.9 \%$ \\
& Stage 2 & 2 & 2 & 28 & 0 & 32 \\
& $0.4 \%$ & $0.4 \%$ & $4.9 \%$ & $0 \%$ & $5.7 \%$ \\
& Stage 3 & 0 & 0 & 0 & 63 & 63 \\
& $0 \%$ & $0 \%$ & $0 \%$ & $11.1 \%$ & $11.1 \%$ \\
& Total & 416 & 51 & 38 & 64 & \\
& $73.1 \%$ & $9.0 \%$ & $6.7 \%$ & $11.3 \%$ &
\end{tabular}

Upper: Number of patients

Lower: Percentage

$A B W$ actual body weight, $A K I$ acute kidney injury, IBW ideal body weight

than the other BMI subgroups $(P=0.033)$, although the severity of illness (i.e., SAPS II and SOFA score) was not significantly different. However, in the AKI [IBW] group, the mortality rate was not significantly different among BMI subgroups $(P=0.736$; Table 3$)$. The risk ratio of mortality in the underweight subgroup of the AKI $[\mathrm{ABW}]$ group was 2.62 (95\% confidence interval $[\mathrm{CI}]$ 1.17-5.32, $P=0.021)$ when compared with the normal subgroup, 4.04 (95\% CI 1.62-9.79, $P=0.0035)$ when compared with the overweight subgroup, and 1.98 (95\% CI $0.77-5.10, P=0.152)$ when compared with the obese subgroup (Table 4).

\section{Relationship between cystatin C and creatinine among the BMI subgroups}

Table 3 shows serum creatinine levels according to the BMI classification in the AKI [ABW] and AKI [IBW] groups. In the AKI [IBW] group, the highest serum creatinine levels significantly increased in line with the BMI (underweight, 1.51 [0.84-2.59]; normal, 2.31 [1.36-4.20]; overweight, 2.56 [1.53-3.60]; and obese, $2.92 \mathrm{mg} / \mathrm{dL}$ [2.10-5.50], $P=0.018)$. However, in the AKI [ABW] group, creatinine levels did not significantly differ across BMI subgroups $(P=0.366)$.

On the other hand, in the AKI $[A B W]$ group, serum cystatin $C$ levels differed significantly across the BMI subgroups $(P=0.036)$. In particular, the underweight and normal subgroups showed significantly higher cystatin $C$ levels than the overweight group (2.78 [2.32-3.64] vs. $1.74[1.01-2.58] \mathrm{ng} / \mathrm{mL}, P=0.023 ; 2.31$ [1.49-3.36] vs. $1.74[1.01-2.58] \mathrm{ng} / \mathrm{mL}, P=0.015$, respectively). However, in the AKI [IBW] group, serum cystatin $\mathrm{C}$ levels did not differ significantly among the BMI subgroups, and they also showed a homogeneous distribution $(P=0.596)$.

\section{Discussion}

Body weight must be taken into consideration in various clinical situations, such as in the management of ARDS and administration of drugs. For example, the low tidal ventilation strategy as a management approach to ARDS is selected based on the IBW, because the lung volume is more closely related to the IBW than the ABW $[17,18]$. In contrast, when calculating the dosage of medications for morbidly obese patients, there is no marked difference in the utility between ABW and IBW [9]. Furthermore, fluid resuscitation is crucial in the management of patients with sepsis, and can increase the body weight by up to $10 \%$ after initial resuscitation [19]. In such cases, it is unclear whether we should determine the baseline body weight at ICU admission or after the patient's condition has stabilized a few hours later. In contrast, IBW can be estimated based on the sex and height, which are not affected by fluid balance. Furthermore, renal function is not affected by changes in temporary variables such as daily body weight. As such, the use of IBW seems logically more suitable for evaluating renal function and establishing a urinary diagnosis of AKI than ABW.

In this retrospective study, we investigated the most appropriate body weight definition required to accurately make a urinary diagnosis of AKI. We found a discrepancy rate of $7.6 \%$ in terms of the urinary diagnosis of AKI between AKI $[\mathrm{ABW}]$ and $\mathrm{AKI}[\mathrm{IBW}]$. The number cases of AKI $[\mathrm{ABW}]$ was higher than AKI [IBW], which meant a higher sensitivity for detecting AKI. In the AKI [ABW] group, the mortality rate was significantly different among the BMI subgroups, while in the AKI [IBW] group, there were no significant differences in the mortality rates among the BMI subgroups. However, there was no difference in the severity of illness among patients diagnosed with AKI based on ABW or IBW. In this regard, the type of body weight might not have clinical significance in terms of the early recognition of urinary AKI, although AKI $[A B W]$ presented higher sensitivity than AKI [IBW].

To date, there is no consensus about what type of body weight should be used to calculate the weight-adjusted hourly urine output for the urinary diagnosis of AKI according to the KDIGO criteria. Only one study has examined this issue [7]; that study found that ABW was more sensitive but less specific than IBW in terms of diagnosing and staging AKI using the urine output criterion. However, the population in that study had a higher mean BMI than our studied population ( 28.0 vs. 22.5 ), and only $7.8 \%$ of patients had a BMI $\leq 20$. Therefore, especially for underweight patients, the utility of ABW to diagnose AKI with regards to its superior sensitivity when compared to IBW remains unclear; our findings suggest that AKI [ABW] might yield more heterogeneity in terms of mortality rate prediction and timing of recognizing AKI than AKI [IBW]. In this study, however, there were no significant differences 


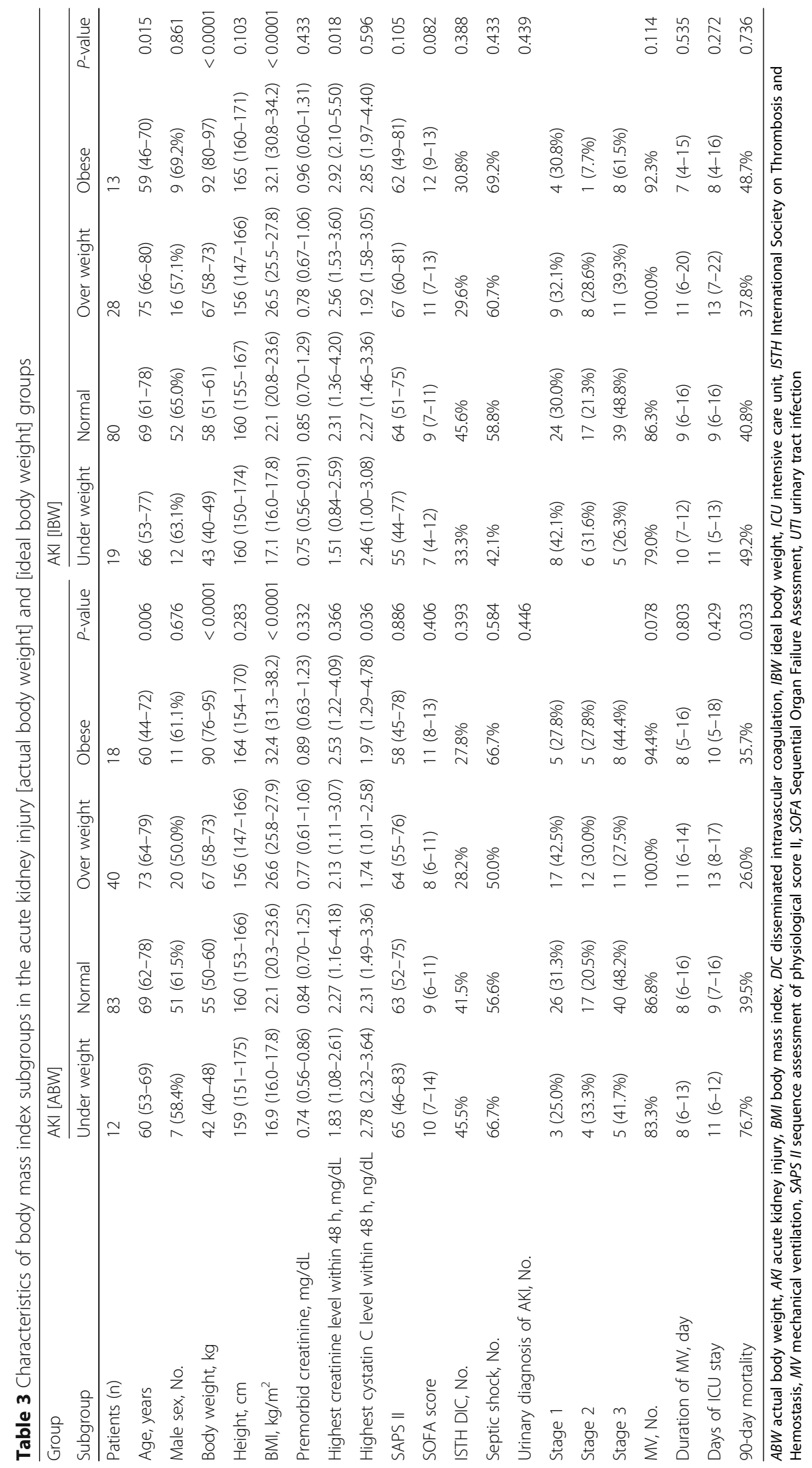


Table 4 Risk ratio for cumulative mortality according to BMI subgroups

\begin{tabular}{|c|c|c|c|c|c|c|c|c|c|}
\hline \multicolumn{5}{|c|}{$\mathrm{AKI}(\mathrm{ABW})$} & \multicolumn{5}{|c|}{ AKI (IBW) } \\
\hline & $\begin{array}{l}\text { Under } \\
\text { weight }\end{array}$ & Normal & $\begin{array}{c}\text { Over } \\
\text { weight }\end{array}$ & Obese & & $\begin{array}{l}\text { Under } \\
\text { weight }\end{array}$ & Normal & $\begin{array}{c}\text { Over } \\
\text { weight }\end{array}$ & Obese \\
\hline \multirow{2}{*}{$\begin{array}{l}\text { Under } \\
\text { weight }\end{array}$} & & $\begin{array}{c}2.62 \\
(1.17-5.32) \\
\end{array}$ & $\begin{array}{c}4.04 \\
(1.62-9.79) \\
\end{array}$ & $\begin{array}{c}1.98 \\
(0.77-5.10)\end{array}$ & \multirow{2}{*}{$\begin{array}{l}\text { Under } \\
\text { weight }\end{array}$} & & $\begin{array}{c}1.48 \\
(0.68-2.92)\end{array}$ & \begin{tabular}{c|c}
1.83 \\
$(0.74-4.62)$ \\
\end{tabular} & $\begin{array}{c}0.97 \\
(0.37-2.68) \\
\end{array}$ \\
\hline & & 0.021 & 0.0035 & 0.152 & & & 0.303 & 0.188 & 0.954 \\
\hline \multirow[t]{2}{*}{ Normal } & & & $\begin{array}{c}1.54 \\
(0.79-3.21) \\
\end{array}$ & $\begin{array}{c}0.76 \\
(0.37-1.69) \\
\end{array}$ & \multirow[t]{2}{*}{ Normal } & & & \begin{tabular}{|c|}
1.24 \\
$(0.61-2.78)$ \\
\end{tabular} & $\begin{array}{c}0.66 \\
(0.31-1.63) \\
\end{array}$ \\
\hline & & & 0.21 & 0.473 & & & & 0.561 & 0.341 \\
\hline \multirow{2}{*}{$\begin{array}{c}\text { Over } \\
\text { weight }\end{array}$} & & & & \begin{tabular}{|c|}
0.49 \\
$(0.20-1.22)$ \\
\end{tabular} & \multirow{2}{*}{$\begin{array}{c}\text { Over } \\
\text { weight }\end{array}$} & & & & $\begin{array}{c}0.53 \\
(0.20-1.49) \\
\end{array}$ \\
\hline & & & & 0.122 & & & & & 0.218 \\
\hline Obese & & & & & Obese & & & & \\
\hline
\end{tabular}

Upper: Risk ratio (95\% confidence interval)

Lower: $P$-value

$A B W$ actual body weight; $A K I$ acute kidney injury; $B M I$ body mass index; IBW ideal body weight

between the two groups in severity of illness. Previous studies suggested that overweight and obese patients presented lower mortality than underweight patients irrespective of the severity of illness [20, 21]. From this perspective, increased mortality in underweight patients with AKI $[\mathrm{ABW}]$ seems more of an "obesity paradox" rather than an underdiagnosis. In this regard, the types of body weight used for urinary AKI might have no clinical significance.

For the early recognition of AKI, 6-h urine output of $<0.5 \mathrm{~mL} / \mathrm{kg} / \mathrm{h}$ and elevated serum creatinine level $(\geq 0.3 \mathrm{mg} / \mathrm{dL}$ ) have been used [3]. Moreover, AKI could be more sensitively detected using both the urine output and creatinine criteria, than using the creatinine criteria alone [4-6, 22]. However, creatinine production is highly heterogeneous across individuals, differs according to muscle mass, physical activity, and dietary meat consumption, and tends to overestimate the prevalence of AKI in obese patients and underestimate the prevalence in underweight patients. In addition, the urinary diagnosis of AKI depends on the type of body weight used. In this context, the present AKI definition might not be ideal for patients with abnormal body weight such as morbidly obese patients. However, serum creatinine levels would easily be elevated in this population because of their high muscle mass, and this phenomenon could help to make an early diagnosis of AKI. Our study supported this speculation: serum creatinine levels increased in line with the BMI. Creatinine shows small variations in underweight patients because of their lower muscle mass than in obese patients, and this might lead to the under-recognition of AKI despite using two different definitions of AKI, creatinine, and urine output criteria.

Measurement of urine output is not only more sensitive and quicker than measuring serum creatinine once a day in detecting AKI, but it is also important to realize that patients only fulfilling AKI definitions based on urine output criteria differ from patients fulfilling AKI definitions based on serum creatinine with or without urine output measurements. In this study, $15.7 \%$ of AKI [ABW] patients and $12.9 \%$ of AKI [IBW] patients were not diagnosed with AKI based on the serum creatinine definition. In this regard, serum creatinine and urine output for diagnosing AKI do not always act complementarily, but may show independent pathophysiologies. Further studies are needed to evaluate this concern in these populations.

To evaluate renal function, serum creatinine was commonly used. However, its value was highly affected by body types. Recently, cystatin $\mathrm{C}$ has alternatively been recommended for the diagnosis of AKI instead of creatinine [23]. Cystatin $C$ is a serum protein filtered freely at the glomerulus as is creatinine, but unlike creatinine, it is produced by all nucleated cells and its level is not determined by muscle mass; therefore, its generation appears to be more uniform across populations [24]. Generally, cystatin $\mathrm{C}$ is not recommended to evaluate renal function, and KDIGO guidelines still use serum creatinine. On the other hand, there are several studies in which cystatin $C$ appears to be a better biomarker in the prediction of AKI, especially in the early phase [25-28]. The concentration of cystatin $C$ peaks earlier than serum creatinine in patients with AKI, and may enable the earlier detection of kidney dysfunction than creatinine [29]. In this study, in line with previous studies, we used cystatin $\mathrm{C}$ to evaluate renal function along with serum creatinine in more detail. As a result, both underweight and overweight patients in the $\mathrm{AKI}[\mathrm{ABW}]$ group showed highly heterogeneous serum cystatin $C$ levels. In this regard, we should recognize that the use of ABW to establish a urinary diagnosis of AKI 
would result in delayed recognition of AKI in the underweight group, compared to the findings obtained using IBW, irrespective of their severity of illness. To confirm this finding, further studies are needed to evaluate the relationship between BMI and cystatin $\mathrm{C}$ concentration in septic AKI patients.

\section{Limitations}

Several limitations associated with the present study should be mentioned. First, this was a single-center, retrospective study. The sample size was relatively small, particularly when divided into BMI subgroups. Further studies are needed to confirm our findings, especially for the underweight patients. Second, we did not evaluate fluid balance before admission to the ICU; therefore, the baseline body weight might differ from that in the premorbid state. In addition, the timing of body weight measurement was not always just after ICU admission. However, we tried to select the most reliable body weight value after ICU admission in an effort to minimize any errors. Third, we did not measure other AKI biomarkers, such as urine neutrophil gelatinase-associated lipocalin (NGAL), to evaluate the accuracy of urinary AKI. Finally, whether or not the patients' underweight statuses were due to malignant disease was unclear. The presence of such disease, for example, cachexia, might be associated with the relatively higher mortality rate observed in this subgroup than that in the other BMI subgroups.

Despite these limitations, our study has several strengths. All of our patients were evaluated using the Sepsis-3 criteria as being at risk for AKI. In addition, to the best of our knowledge, our study is the first to focus on various body weight types in order to evaluate the homogeneity of the timing of establishing a urinary diagnosis of AKI.

\section{Conclusion}

Using $\mathrm{ABW}$ to calculate the weight-adjusted hourly urine output for diagnosing AKI generally increased the sensitivity compared with IBW irrespective of the severity of disease among both groups. This result could be expected due to the increasing number of overweight patients who earlier meet the definition of urinary AKI. Delayed diagnosis, however, was more common among underweight patients in this situation, and clinicians should be cautious when diagnosing urinary AKI using actual body weight.

\section{Abbreviations}

ABW: Actual body weight; AKI: Acute kidney injury; ARDS: Acute respiratory distress syndrome; BMI: Body mass index; eGFR: Estimated glomerular filtration rate; IBW: Ideal body weight; ICU: Intensive care unit; KDIGO: The Kidney Disease Improving Global Outcomes; SAPS-II: Simplified Acute Physiology Score II; SOFA: Sequential Organ Failure Assessment

\section{Acknowledgments}

We appreciate the assistance from the nursing staff of the intensive care unit at Jichi Medical University Hospital, Tochigi, Japan.

\section{Funding}

The study was conducted with help of Jichi Medical University research expense.

Availability of data and materials

The dataset generated and/or analyzed during the current study is not publicly available due to patient-related confidentiality, but it is available from the corresponding author on reasonable request.

\section{Authors' contributions}

SK collected the data, interpreted the statistical results, and wrote the first draft. KK and SN contributed to the design of the protocol and revised the manuscript. YG, KT, TK, JS and MW collected the data and revised the manuscript. All authors are accountable for all aspects of the work and have approved the final manuscript.

\section{Ethics approval and consent to participate}

This study was approved by the Institutional Review Board of Jichi Medical University (Tochigi, Japan) (approval number: 16-116). The need for informed consent was waived on the basis of the study's retrospective observational design preserving the confidentiality of personal information.

\section{Competing interests}

The authors declare that they have no competing interests.

\section{Publisher's Note}

Springer Nature remains neutral with regard to jurisdictional claims in published maps and institutional affiliations.

Received: 7 October 2017 Accepted: 12 April 2018

Published online: 02 May 2018

References

1. Bagshaw SM, George C, Bellomo R, et al. Early acute kidney injury and sepsis: a multicentre evaluation. Crit Care. 2008;12:R47.

2. Kolhe NV, Stevens PE, Crowe AV, et al. Case mix, outcome and activity for patients with severe acute kidney injury during the first 24 hours after admission to an adult, general critical care unit: application of predictive models from a secondary analysis of the ICNARC case mix Programme database. Crit Care. 2008;12(Suppl 1):S2.

3. Kellum JA, Lameire N, Group KAGW. Diagnosis. Evaluation, and management of acute kidney injury: a KDIGO summary (part 1). Crit Care. 2013:17:204.

4. Macedo E, Malhotra R, Bouchard J, et al. Oliguria is an early predictor of higher mortality in critically ill patients. Kidney Int. 2011;80:760-7.

5. Macedo E, Malhotra R, Claure-Del Granado R, et al. Defining urine output criterion for acute kidney injury in critically ill patients. Nephrol Dial Transplant. 2011;26:509-15

6. Prowle JR, Liu YL, Licari E, et al. Oliguria as predictive biomarker of acute kidney injury in critically ill patients. Crit Care. 2011;15(4):R172.

7. Thongprayoon C, Cheungpasitporn W, Akhoundi A, et al. Actual versus ideal body weight for acute kidney injury diagnosis and classification in critically ill patients. BMC Nephrol. 2014;15:176

8. The Acute Respiratory Distress Syndrome Network, Brower RG, Matthay MA Ventilation with lower tidal volumes as compared with traditional tidal volumes for acute lung injury and the acute respiratory distress syndrome. N Engl J Med. 2000;342:1301-8.

9. Erstad BL. Dosing of medications in morbidly obese patients in the intensive care unit setting. Intensive Care Med. 2004;30:18-32.

10. Singer M, Deutschman CS, Seymour CW, et al. The third international consensus definitions for Sepsis and septic shock (Sepsis-3). JAMA. 2016;315:801-10.

11. Devine BJ. Gentamicin therapy. Drug Intell Clin Pharm. 1974;8:650-5.

12. National Kidney Foundation. K/DOQI clinical practice guidelines for chronic kidney disease: evaluation, classification, and stratification. Am J Kidney Dis. 2002;39(2 Suppl 1):S1-266. 
13. Taylor FB Jr, Toh CH, Hoots WK, et al. Towards definition, clinical and laboratory criteria, and a scoring system for disseminated intravascular coagulation. Thromb Haemost. 2001;86:1327-30.

14. Pickering JW, Endre ZH. Back-calculating baseline creatinine with MDRD misclassifies acute kidney injury in the intensive care unit. Clin J Am Soc Nephrol. 2010;5:1165-73.

15. Abhyankar S, Leishear K, Callaghan FM, et al. Lower short- and long-term mortality associated with overweight and obesity in a large cohort study of adult intensive care unit patients. Crit Care. 16(6):R235.

16. Amundson, D. E., Djurkovic, S., Matwiyoff, G. N. The obesity paradox. Crit Care Clin 26(4):583-96

17. Crapo RO, Morris AH, Gardner RM. Reference spirometric values using techniques and equipment that meet ATS recommendations. Am Rev Respir Dis. 1981;123:659-64.

18. Crapo RO, Morris AH, Clayton PD, et al. Lung volumes in healthy nonsmoking adults. Bull Eur Physiopathol Respir. 1982;18:419-25.

19. Balakumar V, Murugan R, Sileanu FE, et al. Both positive and negative fluid balance may be associated with reduced long-term survival in the critically ill. Crit Care Med. 2017;45:e749-57.

20. Wlodzimirow KA, Abu-Hanna A, Slabbekoorn M, et al. A comparison of RIFLE with and without urine output criteria for acute kidney injury in critically ill patients. Crit Care. 2012;16:R200.

21. Le Gall JR, Lemeshow S, Saulnier F. A new simplified acute physiology score (SAPS II) based on a European/north American multicenter study. JAMA. 1993;270:2957-63.

22. Vincent UL, Moreno R, Takala J, et al. The SOFA (Sepsis-related organ failure assessment) score to describe organ dysfunction/failure. On behalf of the working group on Sepsis-related problems of the European Society of Intensive Care Medicine. Intensive Care Med. 1996;22:707-10.

23. Kyhse-Andersen J, Schmidt C, Nordin G, et al. Serum cystatin C, determined by a rapid, automated particle-enhanced turbidimetric method, is a better marker than serum creatinine for glomerular filtration rate. Clin Chem. 1994; 40:1921-6.

24. Shlipak MG, Mattes MD, Peralta CA. Update on cystatin C: incorporation into clinical practice. Am J Kidney Dis. 2013;62:595-603.

25. Odutayo A, Cherney D. Cystatin C and acute changes in glomerular filtration rate. Clin Nephrol. 2012;78:64-75

26. Sjöström $P$, Tidman $M$, Jones I. The shorter $T 1 / 2$ of cystatin $C$ explains the earlier change of its serum level compared to serum creatinine. Clin Nephrol. 2004;62:241-2.

27. Zhang Z, Lu B, Sheng X, Jin N. Cystatin C in prediction of acute kidney injury: a systemic review and meta-analysis. Am J Kidney Dis. 2011;58: $356 e 65$.

28. Yong Z, Pei X, Zhu B, Yuan $\mathrm{H}$, et al. Predictive value of serum cystatin C for acute kidney injury in adults: a meta-analysis of prospective cohort trials. Sci Rep. 2017;23(7):41012.

29. Price JF, Mott AR, Dickerson HA, et al. Worsening renal function in children hospitalized with decompensated heart failure: evidence for a pediatric cardiorenal syndrome? Pediatr Crit Care Med. 2008;9(3):279-84.

\section{Ready to submit your research? Choose BMC and benefit from:}

- fast, convenient online submission

- thorough peer review by experienced researchers in your field

- rapid publication on acceptance

- support for research data, including large and complex data types

- gold Open Access which fosters wider collaboration and increased citations

- maximum visibility for your research: over $100 \mathrm{M}$ website views per year

At BMC, research is always in progress.

Learn more biomedcentral.com/submissions 